\title{
Mapping of lamin A- and progerin-interacting genome regions
}

\author{
Nard Kubben • Michiel Adriaens • Wouter Meuleman • \\ Jan Willem Voncken • Bas van Steensel • Tom Misteli
}

Received: 17 January 2012 /Revised: 23 April 2012 / Accepted: 26 April 2012 / Published online: 19 May 2012

(C) The Author(s) 2012. This article is published with open access at Springerlink.com

\begin{abstract}
Mutations in the A-type lamins A and C, two major components of the nuclear lamina, cause a large group of phenotypically diverse diseases collectively referred to as laminopathies. These conditions often involve defects in chromatin organization. However, it is unclear whether A-type lamins interact with chromatin in vivo and whether aberrant chromatin-lamin interactions contribute to disease. Here, we have used an unbiased approach to comparatively map genome-wide interactions of gene promoters with lamin A and progerin, the mutated lamin $\mathrm{A}$ isoform responsible for the premature aging disorder Hutchinson-Gilford progeria syndrome (HGPS) in mouse cardiac myoytes and embryonic fibroblasts. We find that lamin A-associated genes are predominantly transcriptionally silent and that loss of lamin association leads to the relocation of peripherally localized genes, but not necessarily to
\end{abstract}

Communicated by Erich Nigg

Electronic supplementary material The online version of this article (doi:10.1007/s00412-012-0376-7) contains supplementary material, which is available to authorized users.

\section{N. Kubben $\cdot$ T. Misteli $(\bowtie)$}

Genome Cell Biology Group,

National Cancer Institute, National Institutes of Health,

Bethesda 20892 MD, USA

e-mail: mistelit@mail.nih.gov

M. Adriaens

Department of Experimental Cardiology, AMC Medical Research, Meibergdreef 15,

1100DD, Amsterdam, the Netherlands

W. Meuleman • B. van Steensel

Division of Gene Regulation, Netherlands Cancer Institute,

Amsterdam, the Netherlands

J. W. Voncken

Department of Molecular Genetics, Maastricht University,

6229 ER, Maastricht, the Netherlands their activation. We demonstrate that progerin induces global changes in chromatin organization by enhancing interactions with a specific subset of genes in addition to the identified lamin A-associated genes. These observations demonstrate disease-related changes in higher order genome organization in HGPS and provide novel insights into the role of laminchromatin interactions in chromatin organization.

$\begin{array}{ll}\text { Abbreviations } \\ \text { AIRE } & \text { Autoimmune regulator } \\ \text { ANOVA } & \text { One-way analysis of variance } \\ \text { BAC } & \text { Bacterial artificial chromosome } \\ \text { BPTF } & \begin{array}{l}\text { Bromeodomain and plant homeo-domain } \\ \text { transcription factor }\end{array} \\ \text { ChIP } & \text { Chromatin immunoprecipitation } \\ \text { FDR } & \text { False discovery rate } \\ \text { FPLD } & \text { Familial partial lipodystrophy } \\ \text { FC } & \text { Fold change } \\ \text { GT } & \text { Gene trap } \\ \text { GO } & \text { Gene ontology } \\ \text { GUCE } & \text { GTF2IRD1 upstream control element } \\ \text { HAML } & \text { Human acute myelogenous leukemia factor } \\ \text { HGPS } & \text { Hutchinson-Gilford progeria syndrome } \\ \text { INM } & \text { Inner nuclear membrane } \\ \text { IFN } \gamma & \text { Interferon gamma } \\ \text { LAD } & \text { Lamin-associated domain } \\ \text { LMNA } & \text { LMNA knockout } \\ \text { MEF } & \text { Mouse embryonic fibroblast } \\ \text { NE } & \text { Nuclear envelope } \\ \text { NPC } & \text { Nuclear pore complex } \\ \text { OLFR } & \text { Olfactory receptor } \\ \text { OST-A } & \text { One-STrEP tagged lamin A } \\ \text { OST-P } & \text { One-STrEP tagged progerin } \\ \text { ONM } & \text { Outer nuclear membrane } \\ \text { SF1 } & \text { Steroidogenic factor-1 } \\ & \end{array}$




$\begin{array}{ll}\text { TF } & \text { Transcription factor } \\ \text { TFM } & \text { Transcription factor motif } \\ \text { VMNR } & \text { Vomeronasal receptor } \\ \text { WT } & \text { Wild type } \\ \text { ZF10 } & \text { Zinc finger } 10\end{array}$

\section{Introduction}

The nuclear envelope (NE) defines the boundary between the nucleus and the cytoplasm. The NE is composed of an outer and an inner nuclear membrane (ONM and INM, respectively) interrupted by nuclear pore complexes (NPCs) and is lined by the lamina, a network of the intermediate filaments made up largely of the A-type lamins (lamin A, $\triangle 10, \mathrm{C}$, and $\mathrm{C} 2$ ), encoded by the $L M N A$ gene, and B-type lamins (lamin B1, B2 and B3), encoded by the $L M N B 1$ and $L M N B 2$ genes, respectively (Broers et al. 2006).

Mutations in A-type lamins cause a group of phenotypically diverse diseases, collectively referred to as laminopathies (Broers et al. 2004). They include several types of muscular dystrophies, lipodystrophies, cardiomyopathies, neurological disorders, and premature aging syndromes. In line with the well-established role of A-type lamins in maintaining higher order chromatin organization (Sullivan et al. 1999), laminopathy-associated point mutations in the lamin $\mathrm{A} / \mathrm{C}$ gene (LMNA) often deregulate chromatin structure and organization. The most dramatic laminopathy-associated chromatin reorganization occurs in the premature aging disease Hutchinson-Gilford progeria syndrome (HGPS), where the diseasecausing mutant lamin A isoform progerin causes loss of heterochromatin and reduces mono- and trimethylation of lysines 9 and 20 on histone 3 (Scaffidi and Misteli 2006; Goldman et al. 2004). Another progeria-related lamin A mutation, E145K, leads to alterations in pericentric chromatin, abnormal clustering of centromeres, and mislocalization of telomeres (Taimen et al. 2009). Loss of heterochromatin and mislocalization of HP1 $\beta$ also occur in LMNA mutationmediated mandibuloacral dysplasia (Filesi et al. 2005).

Several lines of evidence suggest that A-type lamins interact directly with chromatin in vivo and that these interactions are affected by $L M N A$ mutations. First, the structurally related B-type lamins directly bind histones $\mathrm{H} 2 \mathrm{~A}$ and H2B in vitro (Goldberg et al. 1999), and lamin B interacts with chromatin in vivo at discrete lamin-associated domains (LADs; de Wit and van Steensel 2009). These domains are characterized by a low gene activity and are demarcated by insulators (9). Second, amino acids 396-430 of the human lamin $\mathrm{A} / \mathrm{C}$ tail bind core histones in vitro (Taniura et al. 1995). Third, the immunoglobulin domain of lamin A/C covalently binds 30-bp dimerized DNA fragments in vitro, and the FPLD-associated R483Q and W mutations lower this affinity (Stierlé et al. 2003). Based on these findings, it was hypothesized that direct interaction of A-type lamins with chromatin is important for chromatin organization and gene regulation (Dechat et al. 2008). While interactions of chromatin with lamin B have been mapped (Peric-Hupkes et al. 2010; Pickersgill et al. 2006), it is unknown which chromatin regions directly interact with A-type lamins in vivo and how loss or mutation of lamin $\mathrm{A} / \mathrm{C}$ affects chromatin organization and gene expression.

To probe the role of the lamina in genome organization, gene expression, and its relevance to laminopathies, we have conducted unbiased, genome-wide mapping of gene promoters that interact with lamin A and/or progerin using a high-affinity pull-down technique (Kubben et al. 2010). We find that lamin A preferentially binds silent or lowly expressed genes. This association facilitates, but does not determine, the peripheral localization, and loss of the interaction is not sufficient for gene activation. Progerin increases lamina-gene interactions by interacting with a specific subset of silent or lowly expressed genes in addition to binding regular lamin Aassociated genes. These data demonstrate a direct and distinct effect of lamin A and progerin on chromatin interaction and organization at the lamina.

\section{Materials and methods}

\section{Cell lines and culture}

An OST-P lentiviral expression plasmid was created by deleting amino acids 609-658 of the pCDH MCSNard One-STrEP tagged lamin A (OST-A) plasmid (Pegoraro et al. 2009). A lentiviral vector expressing shRNA directed against mouse lamin A/C (pSIHpuro-shRNA-mouseLMNA) was generated by annealing 5'gatccGAGCTTGACTTCCAGAAGAACA TttcaagagaATGTTCTTCTGGAAGTCAAGCTCttttg3' and 5'aattcaaaaaGAGCTTGACTTCCAGAAGAACATtctct tgaaATGTTCTTCTGGAAGTCAAGCTCg 3' oligos and subsequent ligation into BamHI- and EcoRI-restricted pSIH H1 plasmid (System Biosciences, Mountain View, USA). Mouse cardiac myocyte NklTAg cells (Rybkin et al. 2003) and mouse embryonic fibroblasts (MEFs) of wild-type and LMNA knockout (LMNA ${ }^{\mathrm{KO}^{-/}-}$) embryos (Sullivan et al. 1999) expressing OST-A or OST-P at expression levels comparable to endogenous lamin A levels were created and cultured as described elsewhere (Kubben et al. 2010).

\section{Western blot}

Cells were lysed in SDS-PAGE Laemmli loading buffer and further denatured by heating for $5 \mathrm{~min}$ at $95^{\circ} \mathrm{C}$. Western blots and immunodetection were performed essentially as 
described elsewhere (Pegoraro et al. 2009). The primary antibodies used for immunodetection were $\alpha$-lamin $\mathrm{A} / \mathrm{C}$ (1:500 dilution, Sc6215; StCruz, Santa Cruz, CA, USA) and $\alpha$-beta actin (1:5,000 dilution, A-5441; Sigma, St. Louis, MO, USA).

\section{Immunofluorescence microscopy}

Immunofluorescence was essentially performed as described elsewhere (Pegoraro et al. 2009). Cells were grown on $0.1 \%$ gelatin/PBS solution-coated multi-well glass slides (MP Biomedicals, Solon, $\mathrm{OH}, \mathrm{USA}$ ), and $\alpha$-LMNA/C (1:500 dilution, Sc-7292; StCruz), $\alpha$-LMNB1 (1:500 dilution, Sc-6217; StCruz), or Chromeo642-conjugated Strep-Tactin and $\alpha$ StrepMAB (both diluted 1:250, IBATAGnology, Göttingen, Germany) were used as a primary antibodies.

\section{Chromatin immunoprecipitation}

NkITAg and MEF cell lines expressing OST-A, OST-P or control vector constructs were formaldehyde fixed ( $1 \%$, $5 \mathrm{~min}$ ), solubilized by sonication in the presence of a high percentage of detergents (1\% SDS, $1 \%$ Trx-100) and diluted to contain equal concentrations of genomic DNA as described previously (Kubben et al. 2010). Samples were end-over-end rotated (overnight, $4^{\circ}$ ) with herring sperm DNA coated Strep-Tactin Matrix (IBA BioTagnology, Göttingen, Germany). Pelleted Strep-Tactin matrix was washed with low and high salt buffer $(0.1 \%$ SDS, $1 \%$ Triton X100, $2 \mathrm{mM}$ EDTA, $20 \mathrm{mM}$ Tris $\mathrm{pH} 8.1,150 \mathrm{mM}$ and $500 \mathrm{mM} \mathrm{NaCl}$ respectively), $\mathrm{LiCl}$ buffer $(0.25 \mathrm{M} \mathrm{LiCl}$, $1 \%$ Igepal-CA630, $1 \%$ deoxycholic acid, $1 \mathrm{mM}$ EDTA, $10 \mathrm{mM}$ Tris $\mathrm{pH} 8.1)$, OST stringent wash buffer $(2 \mathrm{M} \mathrm{NaCl}$, $2 \%$ Trx-100, $500 \mathrm{mM} \mathrm{LiCl}, 0.1 \%$ SDS, $1 \%$ Sodium Deoxycholate, $20 \mathrm{mM}$ Tris $\mathrm{pH}$ 8.1, $2 \mathrm{mM}$ EDTA) and TE buffer (10 mM Tris, $1 \mathrm{mM}$ EDTA pH 8.0). Precipitated protein-chromatin complexes were eluted at room temperature in fresh elution buffer (1\% SDS and $0.1 \mathrm{M} \mathrm{NaHCO} 3)$. After de-cross-linking for $6 \mathrm{~h}$ at $65^{\circ} \mathrm{C}$ in the presence of $200 \mathrm{mM} \mathrm{NaCl}$ and $30 \mu \mathrm{g} / \mathrm{ml}$ RNAase A, DNA was purified using a Qiagen PCR cleanup kit (Qiagen, Hilden, Germany) and amplified according to Whole Genome Amplification kit instructions (Sigma, St. Louis, USA) for further analysis on promoter arrays. Amplified DNA fragments were hybridized to NimbleGen MM8 385 K Refseq promoter arrays, tiling a region of $2000 \mathrm{bp}$ downstream to $500 \mathrm{bp}$ upstream for transcription start sites of $\sim 19,000$ Refseq genes, according to Electronic supplementary material (ESM) Fig. S3. Hybridization and data acquisition were performed inhouse by NimbleGen according to standard procedures (Roche NimbleGen, Madison, WI, USA). To identify OST-A-bound gene promoters in NklTAg cell lines, we compared OST-A chromatin immunoprecipitated DNA vs. control chromatin immunoprecipitated DNA hybridized to the same arrays (ESM Fig. S3) using a within-array analysis approach as specified by NimbleGen (version 6.2; NimbleGen 2010; http://www.nimblegen.com/products/lit/ NG_ChIP-chip_Guide_v6p2.pdf), requiring overlapping peaks between replicates in addition to a false discovery rate (FDR) of $<20 \%$ in each replicate, as successfully applied previously (Romano et al. 2010). Identical to the NkITAG cell line ChIP analysis in MEF cell lines, genes interacting with OST-A or OST-P were identified by a within-array analysis comparing the probe levels of OST-A vs. control chromatin immunoprecipitated DNA or OST-P vs. control chromatin immunoprecipitated DNA hybridized on the same array (ESM Fig. S3). To further test whether the identified OST-A and OST-P targets in MEFs preferentially bind to OST-A or OST-P or interact with equal frequencies to both lamins, we next applied an in-between-array analysis ( $\mathrm{FDR}=5 \%$ ) to directly compare the probe signals of OST-A chromatin immunoprecipitated DNA with OST-P chromatin immunoprecipitated DNA, which were hybridized on separate arrays (ESM Fig. S3). This analysis has been previously successfully applied in ChIP studies and is further described elsewhere (Peric-Hupkes and van Steensel 2010). Both analyses combined led to the identification of three subsets of targets in MEFs: (1) gene promoters that bind preferentially to OST-A (referred to as A targets; OST-A ChIP enriched over OST-P and control ChIP and OST-P ChIP not enriched over control ChIP); (2) gene promoters that bind to both OST-A and OST-P (A\&P targets; OST-A and OST-P ChIP enriched over control ChIP, in which OST-A and OST-P ChIP have comparable levels); (3) or gene promoters that preferentially bind to OST-P (P targets; OST-P ChIP enriched over OST-A and control ChIP and OST-A ChIP not enriched over control ChIP).

Cluster analysis of the identified lamin targets was essentially performed as described previously (Peric-Hupkes and van Steensel 2010). To test whether lamin targets were clustered, we defined the clusters as two or more adjacent laminassociated genes not interrupted by non-target genes. Next, unclustered (cluster size $=1$ gene) and clustered (cluster size $>1$ gene) lamin targets for all chromosomes were compared by Fisher's exact test to the randomly expected occurrences of gene clusters, determined in 10,000 random simulations. Gene ontology (GO) analysis was performed with the DAVID database, comparing gene sets against all annotated gene promoters represented on NimbleGen MM8 385K Refseq promoter arrays, using standard settings for functional annotation clustering and listing significant clusters by the most significant GO biological process classes (Dennis et al. 2003; da Huang et al. 2009). Conservation plots were generated with CEAS analysis (http://cistrome.org/ap/root; Ji et al. 2006), showing the level of conservation of the ChIP regions compared to the genomic background. Genomatix MatInspector 
software (Cartharius et al. 2005) combined with the Genomatix transcription factor motif database (www.genomatix.de) was used to identify enriched transcription factor motifs (TFMs) in target sites and target promoters.

Fluorescent in situ hybridization

To produce probes for DNA fluorescent in situ hybridization (FISH), bacterial artificial chromosomes (BACs; BACPAC Resources Center, Oakland, CA, USA) were labeled by nick translations with dUTP conjugated with biotin or dioxygenin (Roche, Madison, WI, USA) using mouse BAC clones (Acpp, RP24-383K20; Sp100, RP24-235A6; OLFR681, RP24324M2; OLFR1471, RP24-346K11; Eif2b, RP24-285P5; Fanca, RP24-157M4) as described (Meaburn and Misteli 2008). FISH was performed as described elsewhere (Meaburn and Misteli 2008), with the exception of altered denaturation conditions $\left(5 \mathrm{~min}, 85^{\circ} \mathrm{C}\right.$ ) and the introduction of a $45-\mathrm{min}$ $\left(20^{\circ} \mathrm{C}\right)$ incubation step in a block buffer $(3 \% \mathrm{BSA} / 0.05 \%$ Tween-20/4× SSC) diluted (1:50) lamin B antibody (Sc-6217; Santa Cruz Biotechnology, Santa Cruz, CA, USA) prior to incubation with appropriate secondary antibodies.

Using SoftWoRx 3.7.0 Release 13EL (Applied Precision), FISH signals were detected on an IX70 microscope (Olympus) controlled by a Deltavision System (Applied Precision, $\times 601.4$ oil objective lens, auxiliary magnification of 1.5 and optical step size of $0.2 \mu \mathrm{m}$ ) and analyzed in the $z$ section with the brightest signal intensity. The two brightest FISH signals were used for quantification. The frequency of cells with minimally one FISH signal within $500-\mathrm{nm}$ distance of lamin B staining was quantified. Minimally, 150 nuclei were analyzed in duplicate per probe per cell line. Per probe quantification results were statistically tested between different cell lines using the chi-square test. SigmaStat 3.1 software was used for statistical analysis. All test $P$ values $<0.05$ were considered significant.

\section{RNA isolation and expression microarray}

RNA from the cardiac left ventricles of 5-day-old wild-type (WT) and $\mathrm{LMNA}^{\mathrm{GT}-/-}$ mice $(N=2$ each); a LMNA null mouse model (Kubben et al. 2011); and from MEFs infected with a OST-A, OST-P, or empty vector lentivirus $(N=2$ each) were isolated with the RNAeasy minikit (Qiagen, Hilden, Germany) and hybridized to Nugo Mouse Affymetrix Moe430A expression arrays and Affymetrix Mouse Gene 1.0 ST arrays, respectively. Intensity values after hybridization were normalized to the median signal intensity of the array. For individual genes, differences in expression levels were statistically tested by one-way ANOVA. The expression profiles for all sets of targets were statistically analyzed in $\mathrm{R}$ using a two-sample Kolmogorov-Smirnov test between groups, comparing the profiles of the subsets of targets as defined above to one another and against the expression profile of non-targets. Values of $p$ below 0.05 were considered significant. For verification of microarray expression data by PCR, cDNA was synthesized with the iScript $^{\mathrm{TM}}$ cDNA synthesis kit (BioRad, Hercules, CA, USA). SYBR Green real-time quantitative PCR analysis was performed with primers specified in ESM Fig. S4c. Differences in real-time PCR quantification were determined with Student's $t$ test and at the 0.05 significance level.

\section{Results}

Identification of lamin A-associated genes

To map lamin A-interacting chromatin regions at a genomewide scale, we used a recently described high-efficiency pull-down system in which lamin A tagged with the biotin-derived One-STrEP tag (OST-A) is stably expressed at endogenous levels (Kubben et al. 2010) in a murine cardiac myocyte NklTAg cell line (Rybkin et al. 2003). The OST-A fusion protein has previously been characterized in detail (Kubben et al. 2010) and co-localizes in NklTAg cells with endogenous lamin A and lamin B1 (ESM Fig. S1). To identify lamin A-associated genome regions, we performed chromatin immunoprecipitation (ChIP) using the Strep-Tactin matrix, an engineered streptavidin analogue for selective binding $\left(K_{\mathrm{d}}=1 \mu \mathrm{M}\right)$ to the OST tag (Junttila et al. 2005; ESM Fig. S2a). The recovered chromatin was hybridized in duplicate to the NimbleGen promoter tiling arrays covering the 2,500-bp promoter regions flanking the transcriptional start site of $\sim 19,000$ annotated Refseq genes. Chromatin immunoprecipitated DNA from empty vector infected cells was used as a control (ESM Fig. S2a). Six hundred ninety-two lamin A-associated (OST-A) genes were identified based on enrichment in two independent experiments and statistical criteria (Romano et al. 2010; ESM Table S1; see "Materials and methods"). Visual inspection of the processed probe-level data for eight randomly chosen lamin A targets and four non-targets showed little variability between duplicates and confirmed an overall high enrichment of OST-A chromatin immunoprecipitated DNA vs. control chromatin immunoprecipitated DNA in lamin A targets, but not in non-targets.

The 692 OST-A target genes localize to all chromosomes (Fig. 1a and ESM Fig. S5). Of the lamin A targets, $28 \%$ localize in gene clusters (defined as two or more adjacent genes), which is fivefold higher $\left(p<1.0 \times 10^{-4}\right.$; Fisher's exact test) than expected for random gene association (ESM Fig. S6a, b). GO analysis identified the enrichment of lamin A targets in the categories " $G$ protein-coupled receptor signaling" $\left(N=147 / 659\right.$ annotated genes, $p<1.0 \times 10^{-20}$; Fig. 1b), mainly consisting of olfactory receptors (OLFR) 
Fig. 1 Genomic localization and characteristics of laminassociated genes. a ChIP-chip targets of OST-A in NklTAg myocytes plotted according to their genomic location on a relative scale correcting for individual chromosome size differences. b Gene ontology $(G O)$ analysis on identified lamin A targets. Per GO category, the number of lamin Aassociated genes enriched in comparison to the total number of GO annotated genes is indicated, as well as the minimal amount of genes to reach significance $(p=0.05)$
A

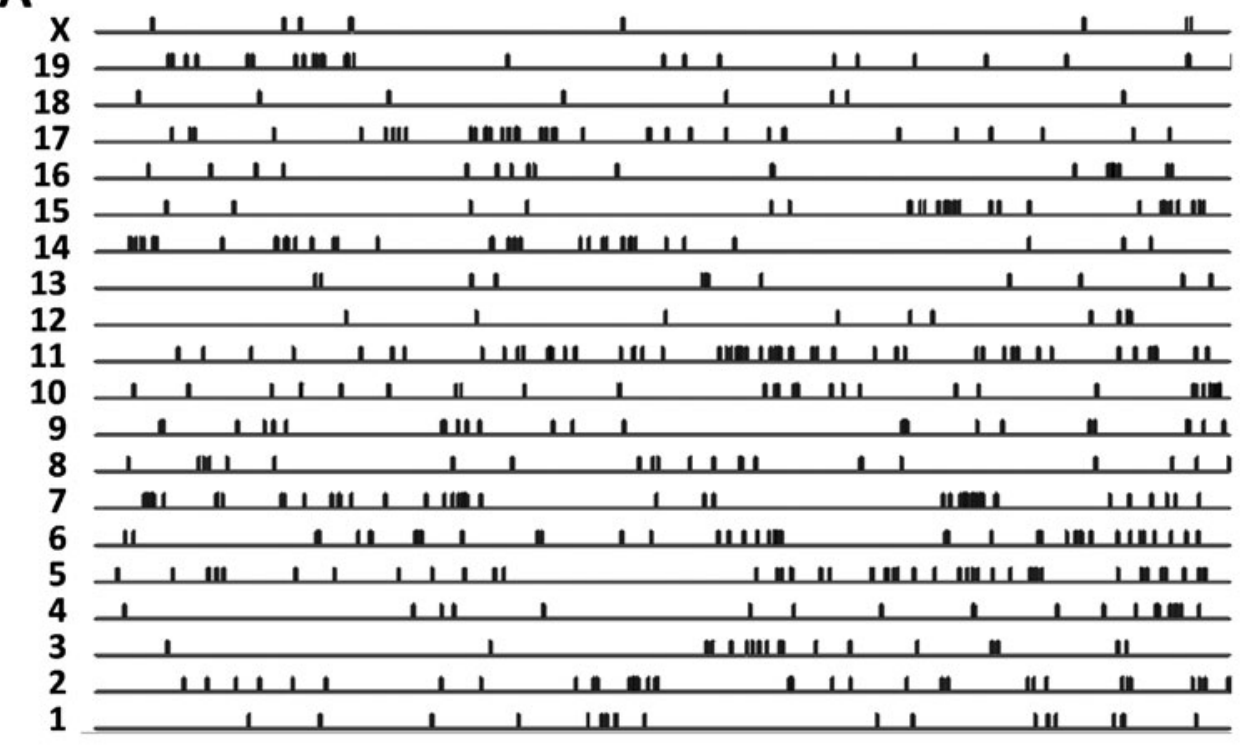

B

\begin{tabular}{lccc} 
Go Category & $\begin{array}{c}\text { N / All } \\
\text { annotated } \\
\text { ChIP targets }\end{array}$ & $\begin{array}{c}\text { N / All } \\
\text { annotated } \\
\text { ChIP targets } \\
\text { for } \mathbf{p}=0.05\end{array}$ & P value \\
\hline \hline G protein coupled receptor signaling & $147 / 659$ & $67 / 659$ & $<1.0 \times 10^{-20}$ \\
Glutamate Receptor Activity & $8 / 659$ & $6 / 659$ & $2.5 \times 10^{-4}$ \\
\hline \hline
\end{tabular}

and VMNR ( $N=104, N=11$ respectively), and "glutamate receptor activity" $\left(N=8 / 659, p=2.5 \times 10^{-4}\right.$; Fig. $\left.1 b\right)$. Conservation plots did not reveal a significant sequence conservation of precipitated DNA fragments (ESM Fig. S7), arguing against the existence of a specific lamin A-binding sequence. Analysis of TFMs revealed 4 of the 181 known TFMs to be significantly enriched in OST-A-associated gene promoters in comparison to random promoter sequences $(p<0.05$; ESM Table S2). These enriched TFMs consist of those for the HAML $\left(p=3.2 \times 10^{-4}\right)$, BPTF $(p=4.3 \times$ $\left.10^{-4}\right)$, AIRE $\left(p<1.0 \times 10^{-4}\right)$, and ZF10 TF families $(p=$ $3.2 \times 10^{-2}$ ). These data demonstrate that A-type lamininteracting gene promoters preferentially localize in genomic clusters, lack unique consensus lamin A-binding sequences, and are enriched for a small set of specific transcription factor binding motifs.

\section{Nuclear localization of lamin A-associated genes}

To assess the subnuclear localization of lamin A-associated genes and to validate the ChIP technique, the location of four randomly chosen lamin A targets and two non-targets were probed by DNA FISH in NkITAg cells. We measured the percentage of cells with at least one FISH signal within $500 \mathrm{~nm}$ of the nuclear periphery identified by lamin B, representing $<5 \%$ of the average nuclear diameter $(10.5 \pm$
$1.5 \mu \mathrm{m}$; Fig. 2a). For the randomly selected lamin A targets Acpp, Sp100, Olfr681, and Olfr1471 located on chromosomes 9, 1, 7, and 19, respectively, at least one allele localized to the periphery in $55,45,55$, and $30 \%$, respectively, of cells compared to 17 and $9 \%$ for the non-targets Eif $2 b$ and Fanca (Fig. 2b). The difference between the peripheral localization of lamin A targets and non-targets is statistically significant at $p<0.001$ in a $\chi^{2}$ test.

Next, we set out to probe the effects of loss of A-type lamins on the subnuclear localization of lamin A-associated genes by knocking down lamin $\mathrm{A} / \mathrm{C}$ in NklTAg cells using shRNA. As a control, the expression of lamin A/C shRNA resulted in over $90 \%$ knockdown for both lamin A and C proteins (ESM Fig. S4b). Upon knockdown, the peripheral localization of the lamin A target loci Acpp, Sp100, and OLFR681 decreased significantly to 35,31 , and $25 \%\left(\chi^{2}\right.$ test: $p<0.01$; Fig. $2 b$ ). In contrast, the least peripherally localized lamin-associated OLFR1471 locus did not change its position upon loss of lamin A ( $\chi^{2}$ test: $p>0.05$; Fig. $2 b$ ). The non-targets Eif $2 b$ and Fanca retained their subnuclear, non-peripheral positions ( $\chi^{2}$ test: $p>0.05$; Fig. $2 b$ ). On the other hand, the overexpression of OST-A at endogenous levels did not affect the localization of lamin A-associated genes, with the exception of the lamin A target OLFR681 (from 46 to $63 \%, p<0.05, \chi^{2}$ test). These findings support a role for lamin $\mathrm{A}$ in retaining gene promoters at the nuclear periphery. 
Fig. 2 Subnuclear localization of lamin A-associated genes. a FISH analysis of one lamin A targets (Sp100, green) and one non-target (Fanca, red) gene in NklTAg cells. Targets are defined as FISH signals overlapping with or within $500 \mathrm{~nm}$ of lamin B staining (blue). The white line indicates a distance of 1,500 nM. b Quantification of FISH signals for four lamin A targets (Acpp, Sp100, Olfr681, and Olfr1471) and two non-targets (Eif2b and Fanca) in NklTAg cells infected with control shRNA, lamin A/C shRNA, OST-A, or a control vector. Asterisks indicate a significant ( $p<0.05, \chi^{2}$ test) change in peripheral positioning in comparison to control infected cells
A Target at nuclear lamina Target not at nuclear lamina

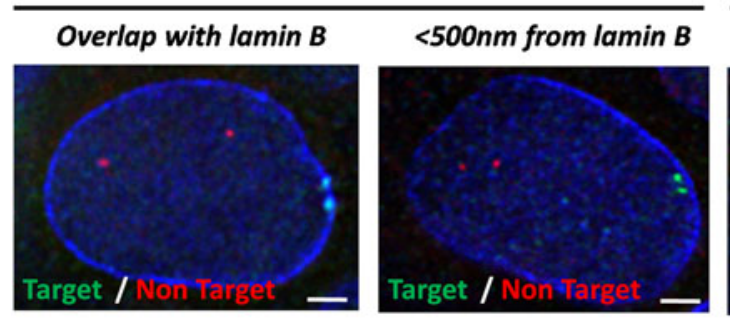
$>500 \mathrm{~nm}$ from lamin B

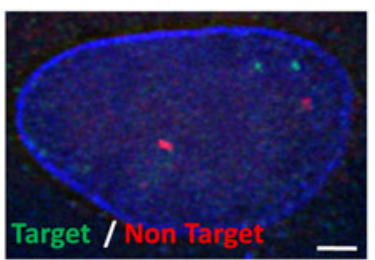

B

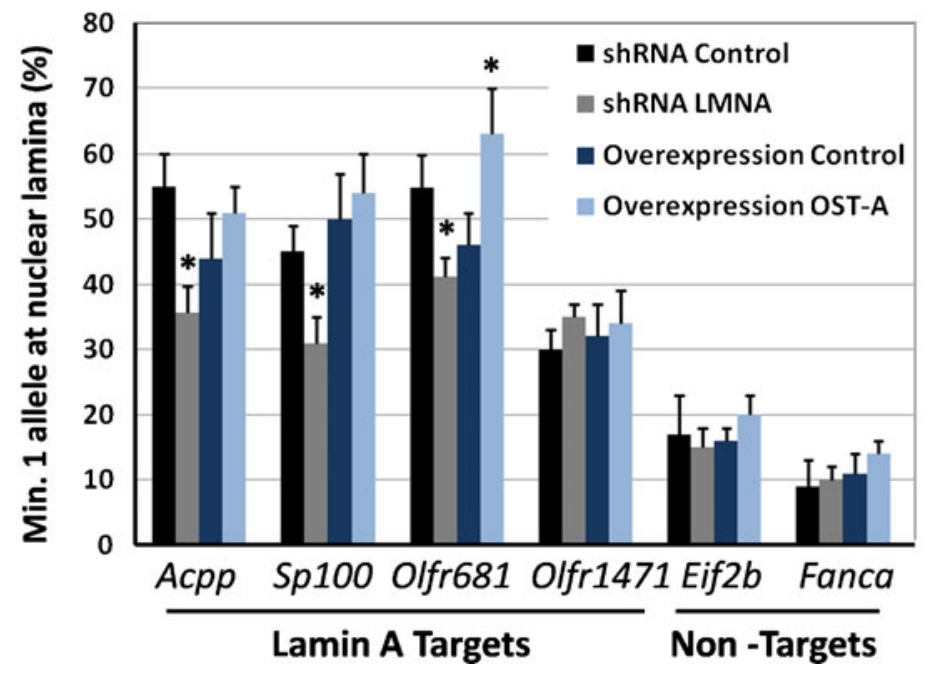

gene GAPDH), 313 genes (56\%) were expressed between the 20th and 80th percentiles $\left(2.57<\log _{2}>6.77\right)$, and 71 genes $(13 \%)$ were expressed above the 80th percentile $\left(\log _{2}>6.77\right.$; ESM Table S3). Highly expressed lamin A interactors $\left(\log _{2}>6.77, N=71\right)$ were not enriched for any GO category. Loss of A-type lamins in LMNA $^{\mathrm{GT}-1-}$ cardiac tissues significantly changed the transcriptional profile of 1,136 genes (Kubben et al. 2011). The average expression fold change (FC) of the 559 lamin A-associated genes was similar to the FC observed in the total genome and indicated no preferential effect of loss of A-type lamins on the lamin A targets (OST-A targets: $\mathrm{FC}=0.99 \pm 0.08, p=0.734$; nontarget: $\mathrm{FC}=1.01 \pm 0.11, p=0.624$, two-sample Kolmogorov-Smirnov test; Fig. 3a). Individual lamin Abound genes that significantly changed expression upon loss of A-type lamins $(N=12)$ were mostly expressed between $\log _{2}$ expressions of 2.57 and 6.77 (20th to 80th percentiles, $N=8$ ), but also included three low expressors $\left(\log _{2}<2.57,20\right.$ th percentile) and repin1, which was expressed highly $\left(\log _{2}=7.30\right.$; ESM Table S4). This group of 12 lamin A targets was not enriched for a particular GO category ( $p>0.05$; ESM Table S4). These findings not only support a role for lamin $\mathrm{A}$ in the nuclear organization of chromatin via capturing transcriptionally silent genes but also suggest that loss of this association is not sufficient for gene activation. 
A

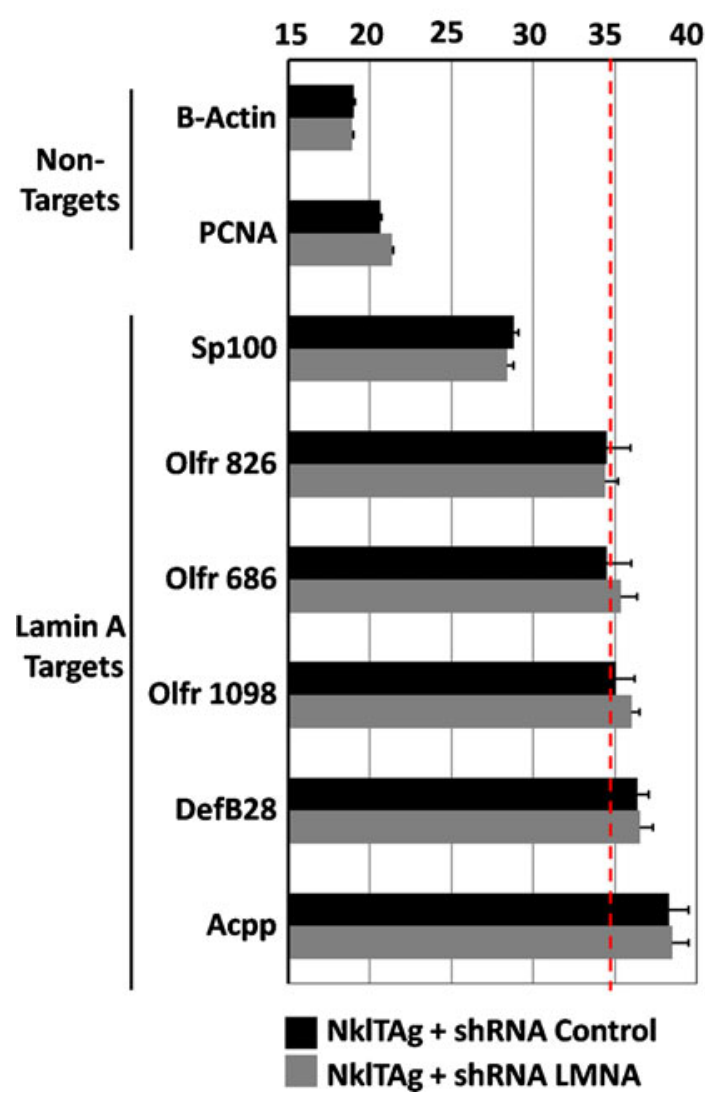

Fig. 3 Expression profiles of lamin A-associated genes. a Real-time quantitative PCR on six lamin A targets (Sp100, Olfr826, olfr686, olfr 1098, Defb28, and Acpp) and two non-targets (B-Actin and Pcna) in the presence and absence of lamin A/C shRNA in NklTAg myocytes. $C_{\mathrm{t}}$ values $( \pm \mathrm{SD})$ were determined in six independent samples using equal amounts of starting material (100 ng cDNA) and corrected

Identification of lamin A- and progerin-associated genome regions

After characterizing lamin A-chromatin interactions in cardiac mycoytes, we next set out to probe for aberrant progerin-chromatin interactions in a separate set of experiments performed in MEFs. To identify genome regions which interact preferentially with lamin A, progerin, or both, we expressed OST-tagged lamin A (OST-A) or progerin (OST-P) at endogenous levels in MEFs (Kubben et al. 2010). OST-A and OST-P co-localize with endogenous A-type lamins and LMNB1 in MEFs (ESM Fig. S1). OST-P expression, in contrast to OST-A, leads to distortions of the nuclear lamina (ESM Figs. S1 and S4a) and global loss of LAP2 and HP1 $\gamma$ nuclear levels (Kubben et al. 2010) similar to the cellular phenotypes observed in HGPS patient cells (Scaffidi and Misteli 2006), further indicating the full functionality of OST-tagged lamin A and progerin in MEFs. Lamin A- and progerin-associated gene promoters were
B
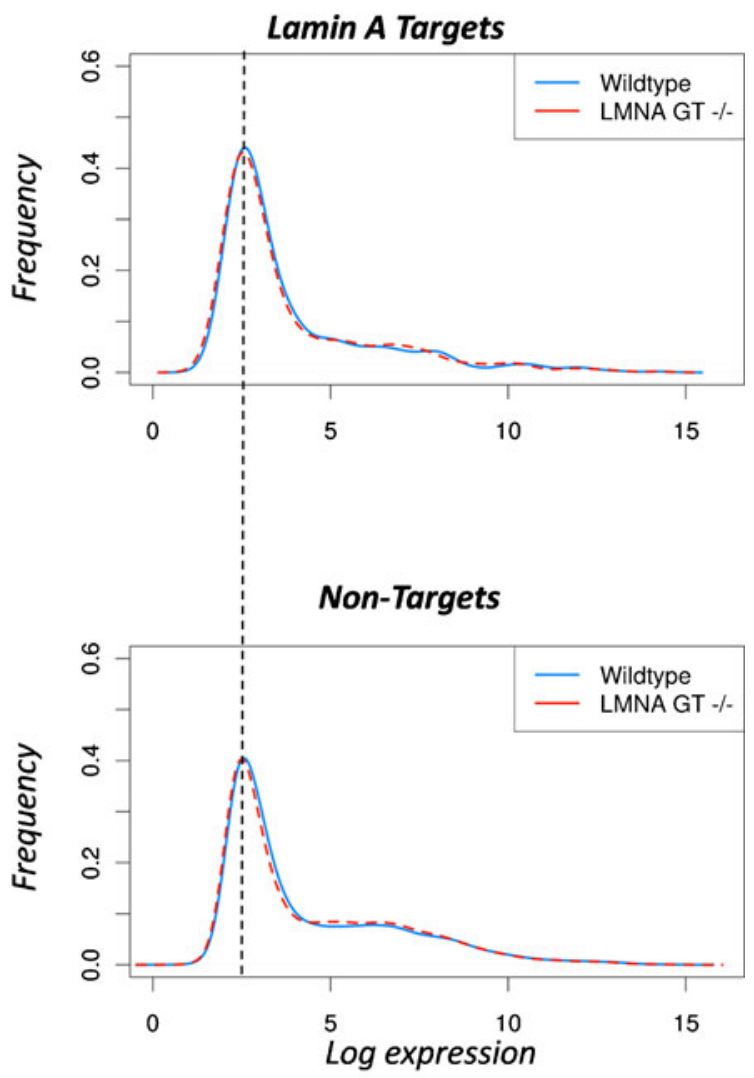

for the mRNA levels of the housekeeping gene GAPDH. The detection limit of quantitative PCR $\left(C_{\mathrm{t}} \sim 35\right)$ is indicated with a dotted line (Akhunova et al. 2009). b Genome-wide RNA expression profiles ( $\log _{2}$ values) of lamin A-associated genes and non-target genes in cardiac left ventricle of wild-type and $\mathrm{LMNA}^{\mathrm{GT}-/-}$ mice, a functional knockout for A-type lamins (Kubben et al. 2011)

identified by a combined statistical analysis (see "Materials and methods") comparing individual array signals from OST-A chromatin immunoprecipitated chromatin and OST-P chromatin immunoprecipitated chromatin with each other and chromatin immunoprecipitated chromatin from control vector infected cells (ESM Fig. S2b). We identified 1,900 lamin A-associated gene promoters and 1991 progerin-associated gene promoters (Fig. 4a and ESM Table S1). Lamin-associated genes identified in NklTAg and MEF cells significantly overlapped as 37 and $38 \%$ of the genes identified at the nuclear lamina in NklTAg cells, associated with lamin A or progerin, respectively, in MEFs as well $\left(p<1 \times 10^{-4}, \chi^{2}\right.$ test $)$.

The vast majority $(99.6 \%, N=1,892)$ of the identified lamin-associated genes in MEFs interacted with both lamin $\mathrm{A}$ and progerin and will further be referred to as A\&P targets (Fig. 4a). With the exception of eight genes (A targets; ESM Table S5), all lamin A-associated genes were also bound by progerin. In contrast, a larger set of 99 genes 
A
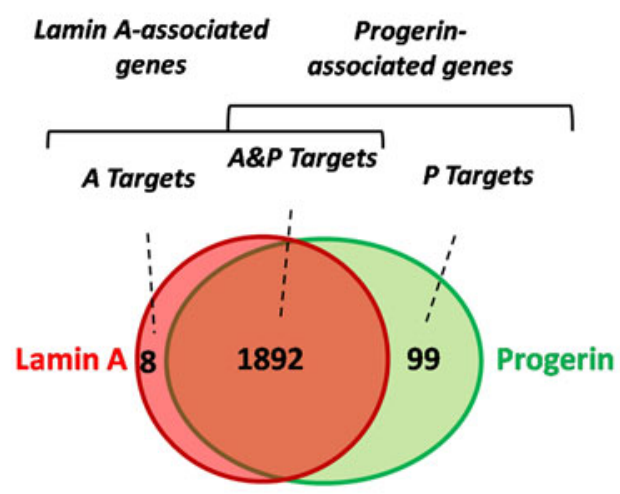

B

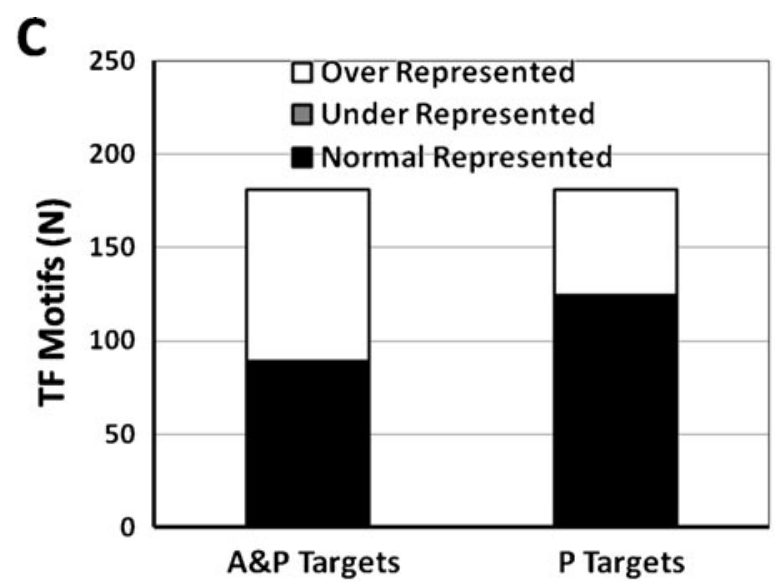

\begin{tabular}{|c|c|c|c|c|}
\hline $\begin{array}{l}\text { CC Target } \\
\text { Genes }\end{array}$ & GO Category & $\begin{array}{c}\mathrm{N} / \text { All } \\
\text { annotated } \\
\text { CCtargets }\end{array}$ & $\begin{array}{c}N / \text { All } \\
\text { annotated } \\
\text { CC targets } \\
\text { for } p=0.05\end{array}$ & $P$ value \\
\hline \multirow{3}{*}{ A\&P Targets } & G protein coupled receptor signaling & $788 / 1418$ & $144 / 1418$ & $<1 \times 10^{-20}$ \\
\hline & Aromatase activity & $14 / 1418$ & $6 / 1418$ & $1.5 \times 10^{-5}$ \\
\hline & Steroid delta-isomerase activity & $3 / 1418$ & $3 / 1418$ & $5.0 \times 10^{-2}$ \\
\hline \multirow{2}{*}{ P Targets } & G protein coupled receptor signaling & $21 / 98$ & 10/192 & $8.6 \times 10^{-4}$ \\
\hline & Endopeptidase activity & $9 / 98$ & $5 / 192$ & $1.5 \times 10^{-3}$ \\
\hline
\end{tabular}

Fig. 4 Characteristics of common and differential lamin A- and progerin-associated genes. a Venn diagram of lamin A- and progerininteracting genes determined by ChIP-chip in MEF cells (ESM Fig. S2). b Gene ontology $(G O)$ analysis on A\&P and $\mathrm{P}$ targets indicating the GO class, the amount of genes $(N)$ per total amount of annotated ChIP target genes, the minimal amount of targets needed for a significance level of 0.05 , and the significance level. c Transcription factor motif analysis on all subclasses of lamin-associated genes for respectively over-, under-, and normal representation of 181 transcription factor motifs $(T F M)$
$(0.5 \%)$ was found to preferentially interact with progerin, but not lamin A (P targets; ESM Table S6). Closer examination of the genomic localization reveals that 76 and $74 \%$ of the lamin A- and progerin-associated genes, respectively, are located in clusters (2-31 adjacent targets), which is more frequent than expected for random gene sets $\left(p<1 \times 10^{-4}\right.$; ESM Fig. S6c-f), and both sets of interactors overlap significantly $\left(p<1 \times 10^{-15}\right.$; Fisher's exact test).

A\&P targets were significantly enriched in " $\mathrm{G}$ proteincoupled receptor signaling" (788 of 1,488 annotated genes, $p<1.0 \times 10^{-20}$; Fig 4b), "aromatase activity" (14 of 1,488, $p=1.5 \times 10^{-5}$; Fig 4b), and "steroid delta-isomerase activity" ( 3 of $1,488, p=5.0 \times 10^{-2}$ ) GO categories. $\mathrm{P}$ targets were to a lesser extent enriched for " $G$ protein-coupled receptor signaling" (21 of 98 annotated genes, $p=8.6 \times 10^{-4}$ ) and also enriched for the "endopeptidase activity" (9 of $98, p=1.5 \times$ $10^{-3}$ ) GO category. The enrichment of different GO families amongst $\mathrm{P}$ and $\mathrm{A} \& \mathrm{P}$ targets suggests distinct preferential association of various gene groups with progerin.
No consensus lamin binding sequence could be identified in P or A\&P targets (ESM Fig. S7). TFM analysis indicated the enrichment of A\&P-associated gene promoters for 92 of 181 TFMs ( $p<0.05$; Fig. 4c and ESM Table S2). The most highly enriched TFMs include those for the hepatocyte nuclear factor $6\left(p<1.0 \times 10^{-10}\right)$, the spermatide-specific transcription factor RUSH $\left(p<1.0 \times 10^{-10}\right)$, the keratinocyte differentiation required transcription factor Brn-5 $(p<1.0 \times$ $\left.10^{-10}\right)$, and neuronal expressed Brn Pou domain TF families (BRNF, $p<1.0 \times 10^{-10}$ ). In contrast, $\mathrm{P}$ targets were less strongly enriched (minimal $\left.p=9.99 \times 10^{-7}\right)$ for fewer TFMs $(N=57)$. Of the 57 TFMs that occurred more frequently than in random promoter sequences, SF1 $\left(p=4.6 \times 10^{-2}\right)$ and craniofacial development involved GTF2IRD1 upstream control element (GUCE, $p=2.4 \times 10^{-2}$ ) were found to be not enriched in A\&P-associated gene promoters. Overall, these data show that lamin A and progerin interact differentially with chromatin and that progerin-associated genes possess distinct features, including their biological function and TFMs. 
Global gene expression profiles of lamin A- and progerinassociated genes

To examine the expression behavior of lamina-associated genes in response to the overexpression of lamin A or progerin, we analyzed mRNA from MEFs transfected with OSTA, OST-P, or a control vector using expression arrays. The introduction of progerin changed the global transcriptional profile significantly (468 genes down, 702 genes up; $p<$ 0.05 , ANOVA), and these changes were different from the overexpression of wild-type lamin A (571 up, 517 down; 379 changed by both OST-A and OST-P). In agreement with previous studies using HGPS patient skin fibroblasts (Csoka et al. 2004), we found specific misregulation of genes involved in extracellular matrix organization and cell cycle control in cells expressing progerin. In MEFs infected with a control vector, the expression profiles of $\mathrm{P}$ and $\mathrm{A} \& \mathrm{P}$ targets were statistically different from non-target genes $(p<0.05$, two-sample Kolmogorov-Smirnov test; Fig. 5b). Both A\&P targets $\left(\Delta \log _{2}=-2.2, p<0.05\right.$, two-sample KolmogorovSmirnov test $)$ and, to a lesser extent, $\mathrm{P}$ targets $\left(\Delta \log _{2}=-1.7\right.$, $p<0.05$, two-sample Kolmogorov-Smirnov test) were repressed compared to non-target genes. The mRNA levels of both classes of interactors differed significantly from each other as well $\left(\Delta \log _{2}=-0.6, p<0.05\right.$, two-sample Kolmogorov-Smirnov test). These basal expression levels of the subclasses did not change significantly upon OST-A or OST-P expression in WT MEFs ( $p>0.05$, two-sample Kolmogorov-Smirnov test; Fig. 5a, b), suggesting that the expression of lamin A or progerin is not sufficient to induce a global change in the gene activity of lamina-associated genes.

\section{Progerin-induced changes in gene expression of lamina-associated genes}

Finally, to identify lamina-associated genes whose expression is specifically misregulated in HGPS, we searched for ChIP targets whose transcription was affected by progerin in MEFs. One hundred eighty-nine of the 1,999 genes associated with lamin A and/or progerin display significantly altered expressions upon the introduction of OST-A or OST-P into MEFs (Table 1). This group of interacting genes $(N=189)$ is particularly enriched for transcriptional regulators $(N=43$, "regulation of transcription" GO category, $p=$ $1.9 \times 10^{-5}$ ) and includes ten zinc finger proteins (MYM-type 2 zinc finger, Zinc finger-64, Zinc finger-157, Zinc finger187, Zinc finger-386, Zinc finger-397, Zinc finger-426, Zinc finger-568, and Zinc finger-760); two members of the SWI/ SNF complex (Smarcc2 and Smarca2); two PPAR transcription factor-related proteins (Ppara and Pparglcb); and welldescribed transcriptional regulators like JunD and Retinoblastoma 1 (Rb1; Table 1).
Sixteen targets out of the group of 189 lamin-interacting genes were considered bona fide candidates for HGPS as they all changed expression upon the introduction of progerin in comparison to MEFs expressing lamin A or empty vector constructs (Table 1). Of these candidates, 12 changed expression in response to progerin only (range $C d 93,+16 \%$; Ppcdc, $-12 \%), 3$ were down-regulated in response to progerin while lamin A increased their expression (Lass6: $+8 \%$ OST-A vs. $-3 \%$ OST-P; Smg 7: $+4 \%$ OST-A vs. $-5 \%$ OST-P; Map $3 k 8$ : $+4 \%$ OST-A vs. $-5 \%$ OST-P), and 1 decreased expression more upon the introduction of progerin as compared to lamin A (Jund1: $-4 \%$ OST-A vs. $-7 \%$ OST-P). The expression levels of 11 candidates remained below the 20th percentile of control MEF transcriptome gene expression levels $\left(\log _{2}<3.31\right)$, while the other five candidates were only slightly more highly expressed (range $=3.52<\log _{2}>3.69$ ). Among the HGPS candidates, Jun1d, Lag1 homolog ceramide synthethase (Lass6), and PPAR $\gamma$ coactivator 1B (Pparg1 Cb) are all involved in transcriptional regulation (GO annotation; Table 1). Overall, these analyses identify lamina-associated genes that are specifically controlled by progerin.

\section{Discussion}

Aberrant chromatin organization is a hallmark of many laminopathies, and disrupted lamin A-chromatin interactions have been suggested to contribute to the etiology of several laminopathies (Dechat et al. 2008). We here map in a genome-wide, unbiased fashion genes which interact with lamin A or with the disease-causing lamin A isoform progerin. We found that lamin A preferentially binds to peripherally localized, genomically clustered, and silent genes and that lamin A and progerin display differential chromatin interaction. Furthermore, we demonstrate that loss or gain of interaction with lamins changes the subnuclear position of several interacting genes, but per se is not sufficient to change their gene expression levels. We speculate that altered gene location may predispose genes for subsequent aberrant regulation.

Recent studies using the DamID method to map lamininteracting genome regions have revealed the importance of lamina-chromatin interactions in chromatin organization and gene regulation by identifying sharply defined LADs across the genome (Guelen et al. 2008; Peric-Hupkes et al. 2010). LADs typically contain gene-poor, low-expressed regions and cell type specifically silenced gene clusters (Shevelyov et al. 2009). In agreement with these studies which were based on the analysis of chromatin interactions with lamin B, we here report, using an independent complementary approach, that lamin A-associated genes are generally silent or expressed at very low levels, genomically clustered, and preferentially localized at the nuclear periphery. Consistent with this, GO 
A

A\&P Targets

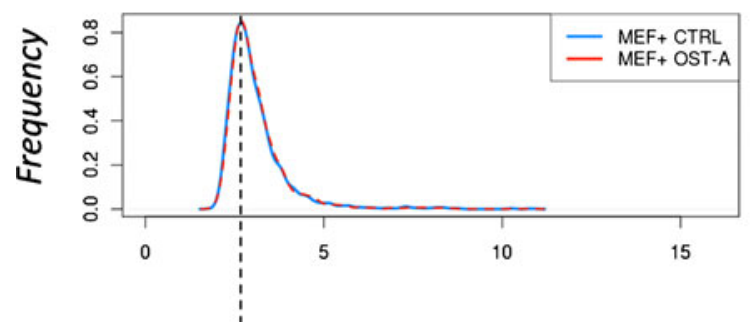

P Targets

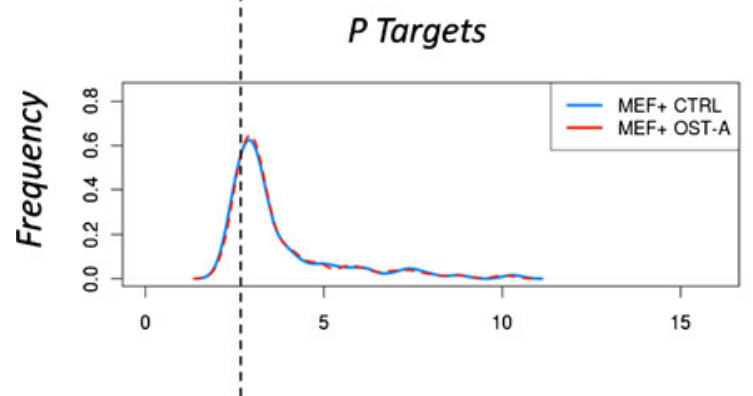

Non-Targets

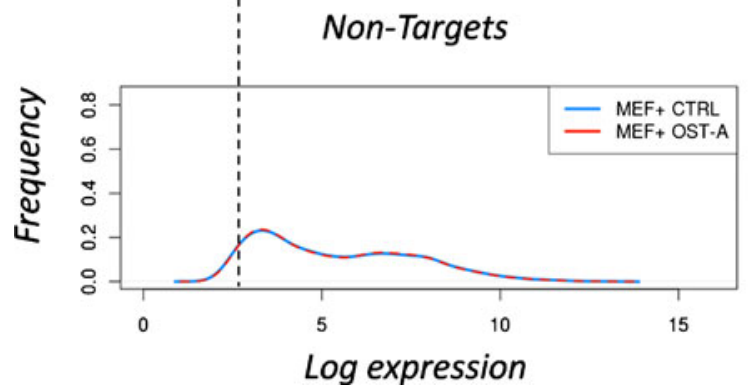

Fig. 5 Expression profiles of lamina/progerin- and progerin-specific associated genes. a Genome-wide RNA expression profiles of A\&P, P, and non-targets in MEFs expressing an empty control vector vs. OST-

analysis reveals that lamin A-associated genes are enriched in gene groups such as sensory perception or glutamate-based neurotransmission, which are non-functional in the fibroblasts or cardiomyocytes analyzed here and hence expected to be transcriptionally silent (Lomvardas et al. 2006; Palmada and Centelles 1998). The enrichment of many tissue and cell typespecific transcription factor binding motifs (TFMs) in lamin A-associated gene promoters further supports this notion. Other enriched TFMs may contribute to the transcriptional repressive environment of the NE by maintaining condensed chromatin (Aoki et al. 1998).

We identified and compared lamin A- and progerinassociated gene promoters. Nearly all lamin A-associated genes $(99.5 \%)$ also interact with progerin. While we cannot completely rule out that the large overlap is due to the crosslinking of endogenous lamin A plus its associated promoter regions to OST-tagged progerin, two lines of evidence argue against this scenario. Firstly, we find that under similar experimental cross-linking conditions, OST-tagged progerin does not pull down NPC components despite the presence of lamin A, which is known to interact with NPCs (Kubben
B A\&P Targets

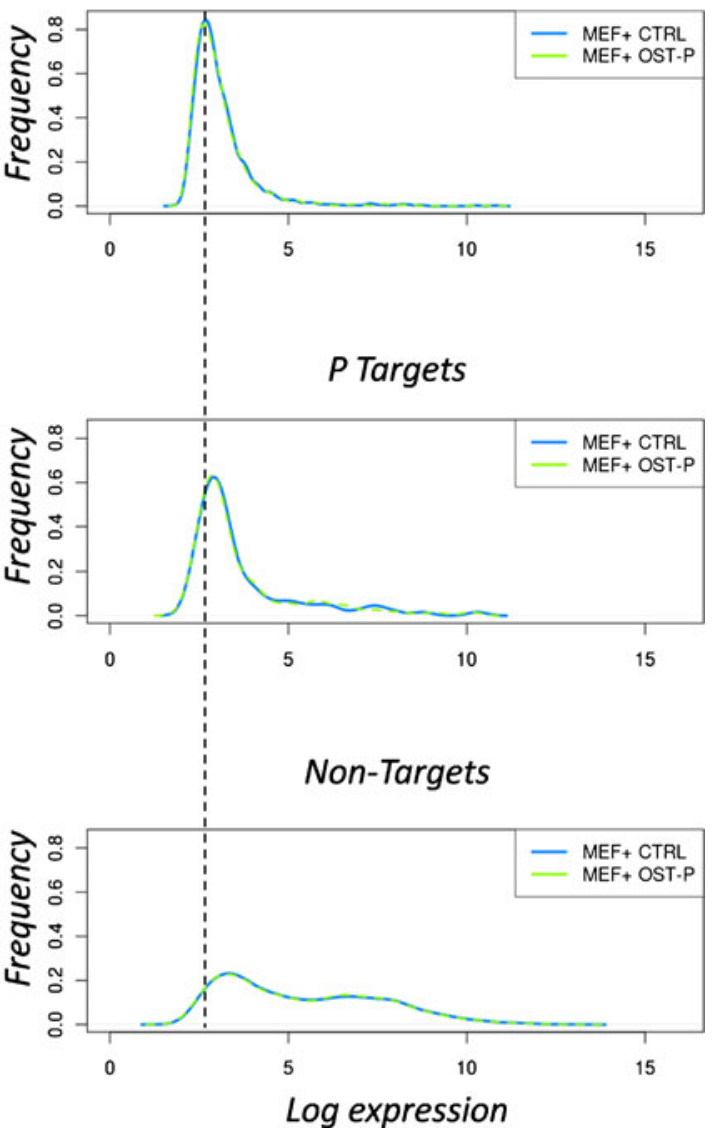

A. b Similar expression profiles in MEFs expressing an empty control vector vs. OST-P

et al. 2010). Secondly, DamID experiments to map laminachromatin interactions performed in the absence of crosslinking using multiple nuclear lamina proteins (lamin B1 and emerin) show a large degree of overlap in the set of interacting genes (de Wit and van Steensel 2009; PericHupkes et al. 2010).

We probed the relevance of lamin-chromatin interactions in the subnuclear positioning and regulation of gene expression in lamin A knockdown and overexpression systems. Despite a substantial relocalization of various lamin A-associated genes to the nuclear interior upon lamin $\mathrm{A} / \mathrm{C}$ knockdown, these loci remain overall more frequently localized at the NE compared to non-targets. The reverse experimental approach by the overexpression of lamin A in cardiac myocytes demonstrates that elevated levels of lamin A can result in even further increased peripheral localization for some lamin A targets. Combined, these findings support a role for A-type lamins in facilitating the recruitment of silenced genes to the nuclear periphery. Interactions with additional nuclear lamina proteins, like emerin and lamin B, which 


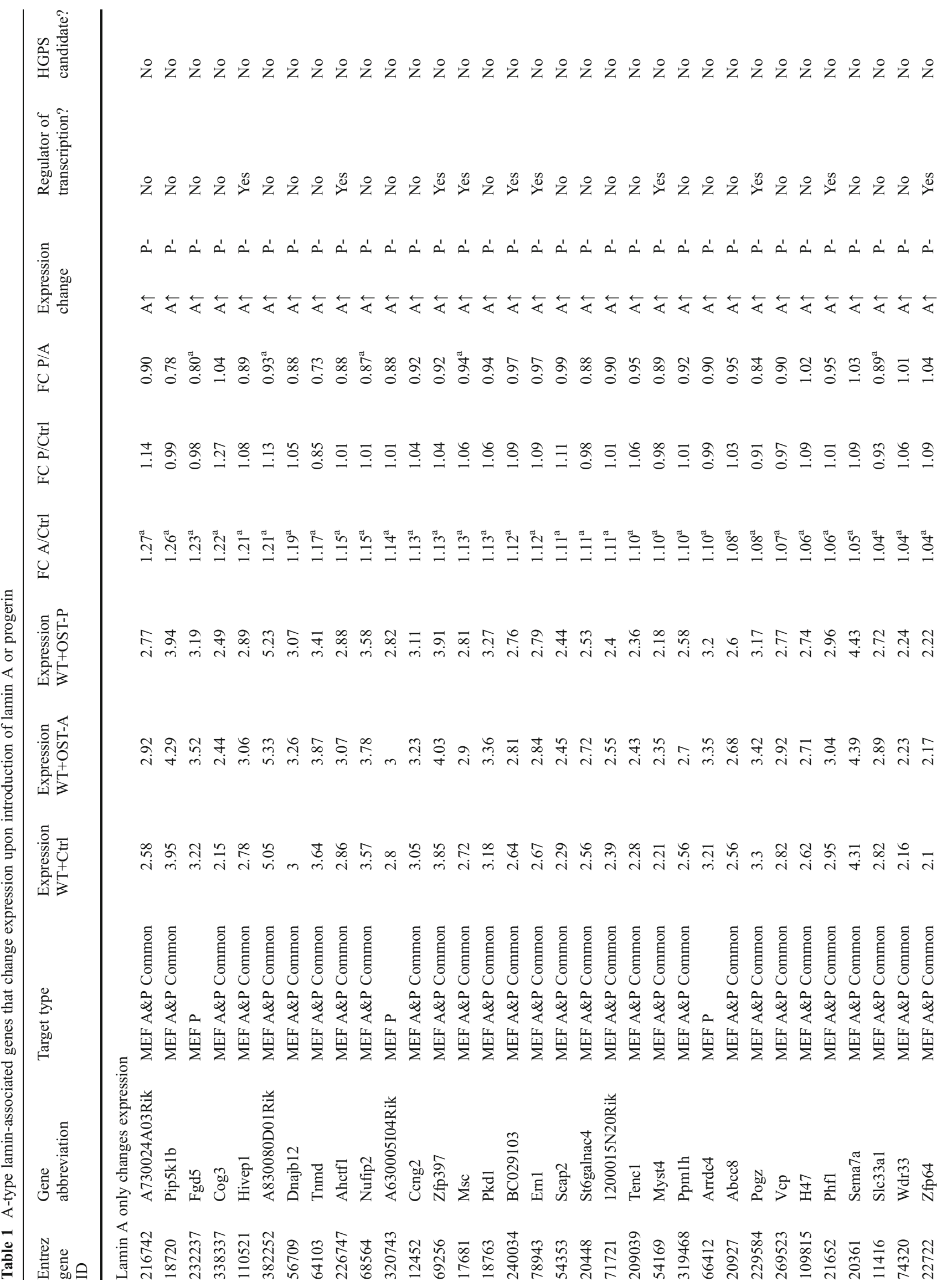




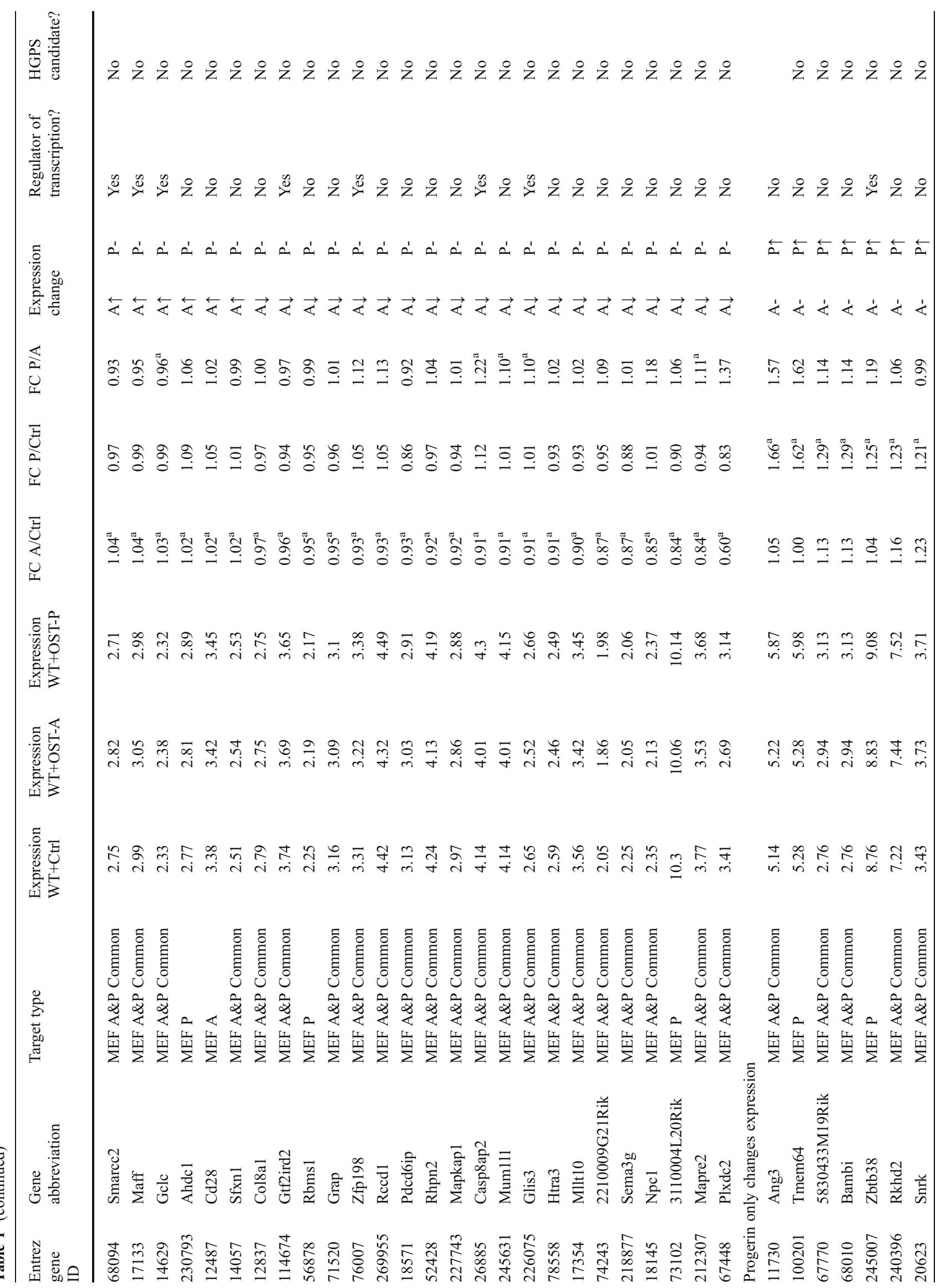




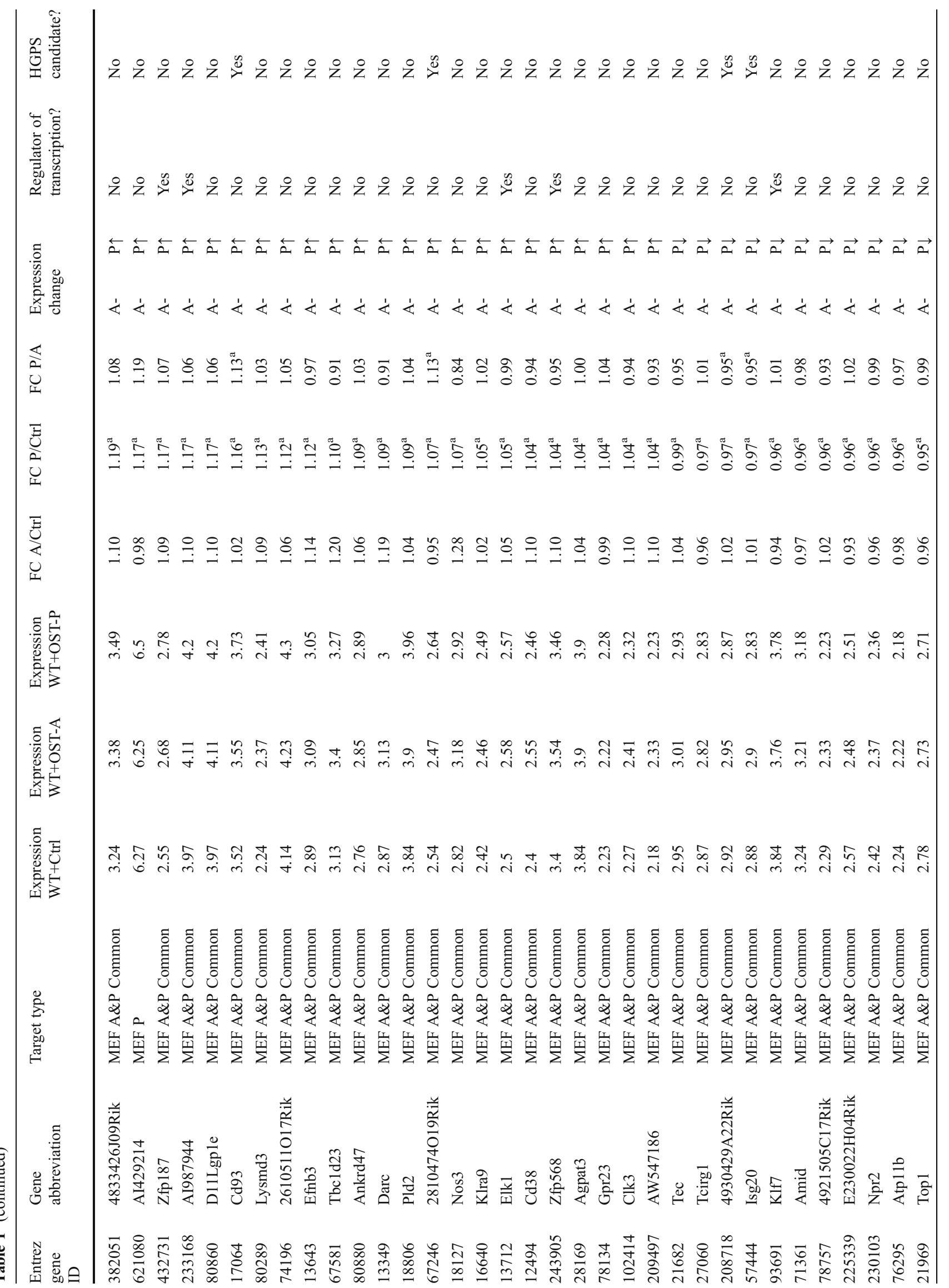




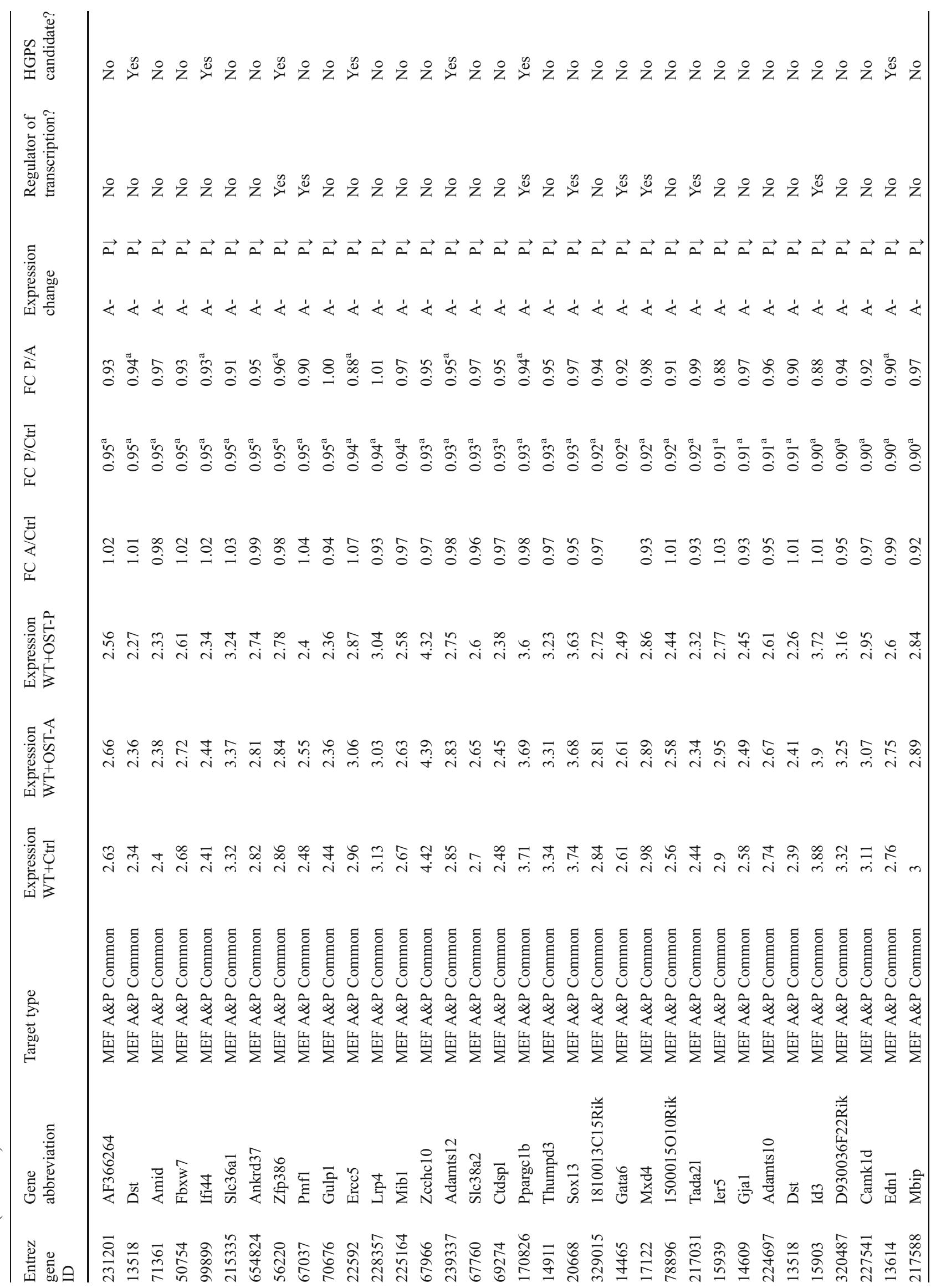




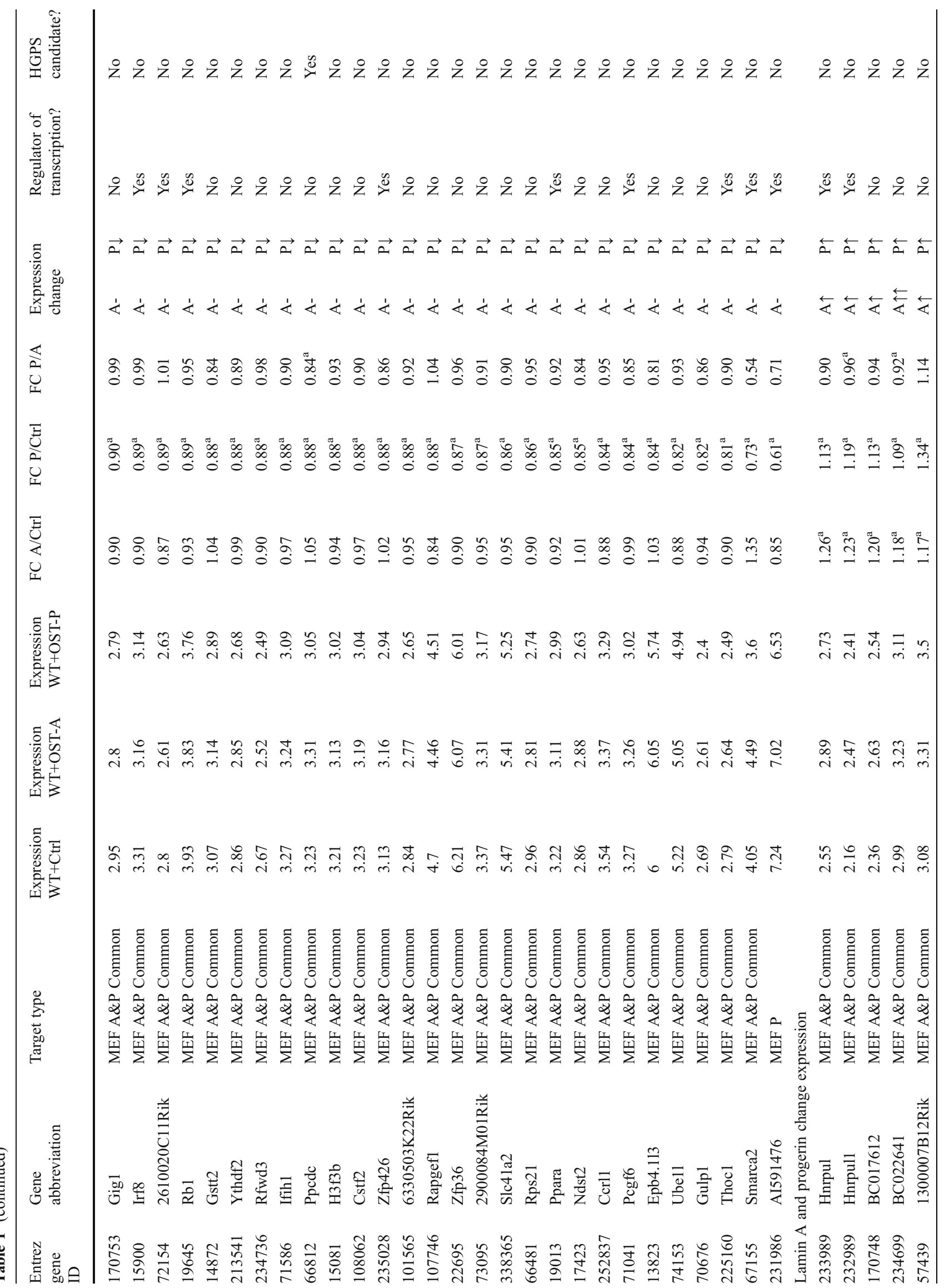




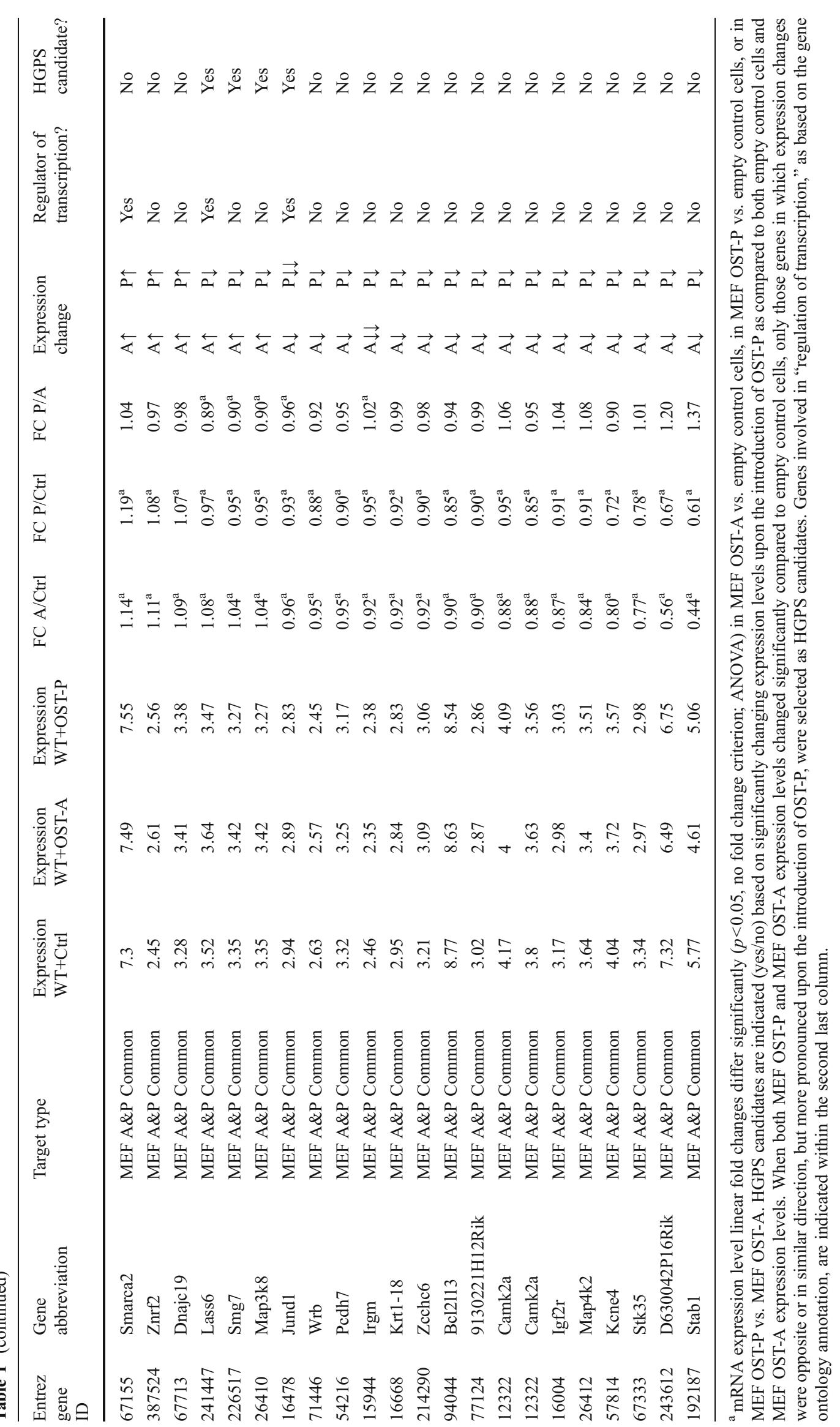


are known chromatin interactors (de Wit and van Steensel 2009), likely contribute to this functional nuclear organization. These findings are in line with the observation that the overexpression of lamin A results in altered gene expression (Scaffidi and Misteli 2011).

A key finding is that while the localization of predominantly silent genes to the periphery is dependent on lamin A, dissociation from the lamina does not necessarily lead to their activation. We speculate that loss of lamina association is only one of multiple steps required for gene activation. Similar behavior has previously been observed for the CFTR locus whose relocation due to the activation and consequent internalization of neighboring genes by itself is insufficient for its activation (Zink et al. 2004; Sadoni et al. 2008). Furthermore, olfactory receptors, which were identified as the most prominent group of lamin A targets in our study, require a multistep process for their stochastic activation (Lomvardas et al. 2006). It is possible that dissociation from the nuclear periphery renders these genes "poised" and facilitates their activation by subsequent signals that may occur, e.g., during differentiation. Extending these studies to differentiation models in which additional factors support the gene activation of specific subsets of target genes will be important.

We identify a group of genes which attach to the lamina only in the presence of progerin. This class of genes is distinct from common lamin A and progerin targets by a number of characteristics: First, progerin targets are involved in different biological processes as compared to common targets, including the general active pathway of proteolysis, and are less enriched for TFMs enriched in promoters bound by both lamin A and progerin. Furthermore, genome-wide expression profile analysis shows that regardless of the presence of progerin, the basal expression levels of common targets are lower than for progerin target genes. These data indicate that transcriptional silencing at the nuclear lamina is strongest for common targets and suggest that progerin targets partially escape a repressive effect of the NE, possibly due to a loss of interaction with the lamina under normal conditions. Despite the fact that these progerin-sensitive genes remain lowly expressed and do not change expression dramatically upon the presence of progerin, additional steps might be required for gene activation in disease situations. A particular enrichment for transcriptional regulators amongst lamin interactors whose expression is differentially regulated by lamin A and progerin hints at a role of general transcriptional misregulation in HGPS, which is in line with previous findings indicating widespread misregulation (Csoka et al. 2004). Among the targets for HGPS, the transcription factor JunD is of particular interest as it has been previously linked to senescence-associated growth arrest (Meixner et al. 2010; Sheerin et al. 2002). Further studies will, however, be required to prove and characterize a role of JunD, as well as the other progerin targets identified here, in HGPS.

Acknowledgments We thank K. Meaburn, T. Karpova, O. Hakim, E. Olson, L. de Windt, A. van Erk, L. Eijssen, R. van Leeuwen, L. van Opstal, and C. Calis for help with experiments and reagents. This work was supported by the Intramural Research Program of the National Institutes of Health (NIH), NCI, Center for Cancer Research; the Dutch Heart Foundation; ZonMW; and the EU-KP7 grant "Inheritance".

Conflict of interest The authors declare to have no competing financial interests or other conflicts of interest.

Open Access This article is distributed under the terms of the Creative Commons Attribution License which permits any use, distribution, and reproduction in any medium, provided the original author(s) and the source are credited.

\section{References}

Akhunova A, Arbieva Z, Grove D, Kubista M, Shipley G (2009) PCR troubleshooting and optimization: the essential guide. In: Kennedy S, Oswald N (eds) Realtime PCR tech guide. A troubleshooting guide: experts give their advice on how to conduct realtime PCR. Caister Academic Press, Norwich

Aoki K, Meng G, Suzuki K, Takashi T, Kameoka Y, Nakahara K, Ishida R, Kasai M (1998) RP58 associates with condensed chromatin and mediates a sequence-specific transcriptional repression. J Biol Chem 273(41):26698-26704

Broers JL, Hutchison CJ, Ramaekers FC (2004) Laminopathies. J Pathol 204(4):478-488

Broers JL, Ramaekers FC, Bonne G, Yaou RB, Hutchison CJ (2006) Nuclear lamins: laminopathies and their role in premature ageing. Physiol Rev 86(3):967-1008

Cartharius K, Frech K, Grote K, Klocke B, Haltmeier M, Klingenhoff A, Frisch M, Bayerlein M, Werner T (2005) MatInspector and beyond: promoter analysis based on transcription factor binding sites. Bioinformatics 21(13):2933-2942

Csoka AB, English SB, Simkevich CP, Ginzinger DG, Butte AJ, Schatten GP, Rothman FG, Sedivy JM (2004) Genome-scale expression profiling of Hutchinson-Gilford progeria syndrome reveals widespread transcriptional misregulation leading to mesodermal/mesenchymal defects and accelerated atherosclerosis. Aging Cell 3(4):235-243

da Huang W, Sherman BT, Lempicki RA (2009) Systematic and integrative analysis of large gene lists using DAVID bioinformatics resources. Nat Protoc 4(1):44-57

de Wit E, van Steensel B (2009) Chromatin domains in higher eukaryotes: insights from genome-wide mapping studies. Chromosoma 118(1):25-36

Dechat T, Pfleghaar K, Sengupta K, Shimi T, Shumaker DK, Solimando L, Goldman RD (2008) Nuclear lamins: major factors in the structural organization and function of the nucleus and chromatin. Genes Dev 22(7):832-853

Dennis G Jr, Sherman BT, Hosack DA, Yang J, Gao W, Lane HC, Lempicki RA (2003) DAVID: database for annotation, visualization, and integrated discovery. Genome Biol 4(5):P3 
Filesi I, Gullotta F, Lattanzi G, D’Apice MR, Capanni C, Nardone AM, Columbaro M, Scarano G, Mattioli E, Sabatelli P, Maraldi NM, Biocca S, Novelli G (2005) Alterations of nuclear envelope and chromatin organization in mandibuloacral dysplasia, a rare form of laminopathy. Physiol Genomics 23(2):150-158

Goldberg M, Harel A, Brandeis M, Rechsteiner T, Richmond TJ, Weiss AM, Gruenbaum Y (1999) The tail domain of lamin Dm0 binds histones H2A and H2B. Proc Natl Acad Sci USA 96:2852-2857

Goldman RD, Shumaker DK, Erdos MR, Eriksson M, Goldman AE, Gordon LB, Gruenbaum Y, Khuon S, Mendez M, Varga R, Collins FS (2004) Accumulation of mutant lamin A causes progressive changes in nuclear architecture in Hutchinson-Gilford progeria syndrome. Proc Natl Acad Sci U S A 101(24):8963-8968

Guelen L, Pagie L, Brasset E, Meuleman W, Faza MB, Talhout W, Eussen BH, de Klein A, Wessels L, de Laat W, van Steensel B (2008) Domain organization of human chromosomes revealed by mapping of nuclear lamina interactions. Nature 453(7197):948-951

Ji X, Li W, Song J, Wei L, Liu XS (2006) CEAS: cis-regulatory element annotation system. Nucleic Acids Res 34 (Web Server issue):W551-W554

Junttila MR, Saarinen S, Schmidt T, Kast J, Westermarck J (2005) Single-step Strep-tag purification for the isolation and identification of protein complexes from mammalian cells. Proteomics 5 (5):1199-1203

Kubben N, Voncken JW, Demmers J, Calis C, van Almen G, Pinto Y, Misteli T (2010) Identification of differential protein interactors of lamin A and progerin. Nucleus 1(6):513-525

Kubben N, Voncken JW, Konings G, van Weeghel M, van den Hoogenhof MM, Gijbels M, van Erk A, Schoonderwoerd K, van den Bosch B, Dahlmans V, Calis C, Houten SM, Misteli T, Pinto YM (2011) Postnatal myogenic and adipogenic developmental: defects and metabolic impairment upon loss of A-type lamins. Nucleus 2(3):195-207

Lomvardas S, Barnea G, Pisapia DJ, Mendelsohn M, Kirkland J, Axel R (2006) Interchromosomal interactions and olfactory receptor choice. Cell 126(2):403-413

Meaburn KJ, Misteli T (2008) Locus-specific and activity-independent gene repositioning during early tumorigenesis. J Cell Biol 180 (1):39-50

Meixner A, Karreth F, Kenner L, Penninger JM, Wagner EF (2010) Jun and JunD-dependent functions in cell proliferation and stress response. Cell Death Differ 17(9):1409-1419

NimbleGen (2010) NimbleGen arrays user's guide, version 6.2. http:// www.nimblegen.com/products/lit/NG_ChIP-chip_Guide_v6p2.pdf

Palmada M, Centelles JJ (1998) Excitatory amino acid neurotransmission. Pathways for metabolism, storage and reuptake of glutamate in brain. Front Biosci 3:d701-d718

Pegoraro G, Kubben N, Wickert U, Gohler H, Hoffmann K, Misteli T (2009) Ageing-related chromatin defects through loss of the NURD complex. Nat Cell Biol 11(10):1261-1267

Peric-Hupkes D, van Steensel B (2010) Role of the nuclear lamina in genome organization and gene expression. Cold Spring Harb Symp Quant Biol 75:517-524
Peric-Hupkes D, Meuleman W, Pagie L, Bruggeman SW, Solovei I, Brugman W, Graf S, Flicek P, Kerkhoven RM, van Lohuizen M, Reinders M, Wessels L, van Steensel B (2010) Molecular maps of the reorganization of genome-nuclear lamina interactions during differentiation. Mol Cell 38(4):603-613

Pickersgill H, Kalverda B, de Wit E, Talhout W, Fornerod M, van Steensel B (2006) Characterization of the Drosophila melanogaster genome at the nuclear lamina. Nat Genet 38(9):1005-1014

Romano A, Adriaens M, Kuenen S, Delvoux B, Dunselman G, Evelo C, Groothuis P (2010) Identification of novel ER-alpha target genes in breast cancer cells: gene- and cell-selective co-regulator recruitment at target promoters determines the response to 17betaestradiol and tamoxifen. Mol Cell Endocrinol 314(1):90-100

Rybkin II, Markham DW, Yan Z, Bassel-Duby R, Williams RS, Olson EN (2003) Conditional expression of SV40 T-antigen in mouse cardiomyocytes facilitates an inducible switch from proliferation to differentiation. J Biol Chem 278(18):15927-15934

Sadoni N, Targosz BS, Englmann A, Fesser S, Koch J, Schindelhauer D, Zink D (2008) Transcription-dependent spatial arrangements of CFTR and conserved adjacent loci are not conserved in human and murine nuclei. Chromosoma 117(4):381-397

Scaffidi P, Misteli T (2006) Lamin A-dependent nuclear defects in human aging. Science 312(5776):1059-1063

Scaffidi P, Misteli T (2011) In vitro generation of human cells with cancer stem cell properties. Nat Cell Biol 13(9):1051-1061

Sheerin A, Thompson KS, Goyns MH (2002) Altered composition of the AP-1 transcription factor in immortalized compared to normal proliferating cells. Cancer Lett 177(1):83-87

Shevelyov YY, Lavrov SA, Mikhaylova LM, Nurminsky ID, Kulathinal RJ, Egorova KS, Rozovsky YM, Nurminsky DI (2009) The B-type lamin is required for somatic repression of testis-specific gene clusters. Proc Natl Acad Sci U S A 106(9):3282-3287

Stierlé V, Couprie J, Östlund C, Krimm I, Zinn-Justin S, Hossenlopp P, Worman HJ, Courvalin J-C, Duband-Goulet I (2003) The carboxyl-terminal region common to lamins $\mathrm{A}$ and $\mathrm{C}$ contains a DNA binding domain. Biochemistry 42:4819-4828

Sullivan T, Escalante-Alcalde D, Bhatt H, Anver M, Bhat N, Nagashima K, Stewart CL, Burke B (1999) Loss of A-type lamin expression compromises nuclear envelope integrity leading to muscular dystrophy. J Cell Biol 147(5):913-920

Taimen P, Pfleghaar K, Shimi T, Moller D, Ben-Harush K, Erdos MR, Adam SA, Herrmann H, Medalia O, Collins FS, Goldman AE, Goldman RD (2009) A progeria mutation reveals functions for lamin A in nuclear assembly, architecture, and chromosome organization. Proc Natl Acad Sci U S A 106:20788-20793

Taniura H, Glass C, Gerace L (1995) A chromatin binding site in the tail domain of nuclear lamins that interacts with core histones. J Cell Biol 131:33-44

Zink D, Amaral MD, Englmann A, Lang S, Clarke LA, Rudolph C, Alt F, Luther K, Braz C, Sadoni N, Rosenecker J, Schindelhauer D (2004) Transcription-dependent spatial arrangements of CFTR and adjacent genes in human cell nuclei. J Cell Biol 166(6):815-825 